\section{P02.03 MICROWAVE ABLATION ENHANCES TUMOR-SPECIFIC IMMUNE RESPONSE IN PATIENTS WITH HEPATOCELLULAR CARCINOMA}

${ }^{1} \mathrm{E}$ Staib* ${ }^{1,2} \mathrm{~K}$ Leuchte, ${ }^{1} \mathrm{M}$ Thelen, ${ }^{1,2} \mathrm{P}$ Gödel, ${ }^{3} \mathrm{~A}$ Lechner, ${ }^{4} \mathrm{P}$ Zentis, ${ }^{1} \mathrm{M}$ Garcia-Marquez, ${ }^{5} \mathrm{D}$ Waldschmidt, ${ }^{1,6} \mathrm{RR}$ Datta, ${ }^{6} \mathrm{R}$ Wahba, ${ }^{7} \mathrm{C}$ Wybranski, ${ }^{2} \mathrm{~T}$ Zander, ${ }^{8} \mathrm{~A}$ Quaas, ${ }^{8} \mathrm{U}$ Drebber, ${ }^{6} \mathrm{DL}$ Stippel, ${ }^{6} \mathrm{C}$ Bruns, ${ }^{1} \mathrm{~K}$ Wennhold, ${ }^{9,10} \mathrm{M}$ von Bergwelt-Baildon, ${ }^{1,6} \mathrm{HA}$ Schlösser. ${ }^{1} \mathrm{Center}$ for Molecular Medicine Cologne, University of Cologne, Köln, Germany; ${ }^{2}$ Department I of Internal Medicine and Center for Integrated Oncology (CIO) Aachen Bonn Cologne Duesseldorf, University Hospital Cologne, Köln, Germany; ${ }^{3}$ Department of Otorhinolaryngology, Head and Neck Surgery, University Hospital, LMU Munich, Munich, Germany; ${ }^{4}$ Cluster of Excellence in Aging-Associated Disease, Core Facility Imaging, University of Cologne, Köln, Germany; ${ }^{5}$ Department of Gastroenterology and Hepatology, University Hospital Cologne, Köln, Germany; ${ }^{6}$ Department of General, Visceral and Cancer Surgery, University Hospital Cologne, Köln, Germany; ${ }^{7}$ Department of Diagnostic and Interventional Radiology, University Hospital Cologne, Köln, Germany; ${ }^{8}$ Institute of Pathology, University Hospital Cologne, Köln, Germany; ${ }^{9}$ German Cancer Consortium (DKTK), Heidelberg, Heidelberg, Germany; ${ }^{10}$ Department of Internal Medicine III, University Hospital, LMU Munich, Munich, Germany

\subsection{6/jitc-2020-ITOC7.39}

Background Thermal ablative therapies, such as microwave ablation (MWA) or radiofrequency ablation (RFA), are standard treatments for HCC. In addition to the local tumor destruction, abscopal effects (a reduction of a tumor mass in areas that were not included in the thermal ablation) could be observed. These systemic effects may be mediated by anti-tumor immune response, which has been described for RFA. MWA is rapidly replacing RFA, but systemic immunostimulatory effects of MWA treatment have been poorly studied.

Materials and Methods Patients receiving MWA for localized HCC were included in this study. Effects of MWA on peripheral blood mononuclear cells (PBMC) of HCC patients treated with MWA were analyzed by multicolor flow cytometry. Tumor-specific immune responses against 7 shared tumor antigens were analyzed using peptide pools in 3-color Fluorospot assays (Interferon-y/Interleukin-5/Interleukin-10). The impact of type, density and localization of tumor-infiltrating lymphocytes was assessed by immunohistochemistry (IHC) of CD3, CD4, CD8, FoxP3, CD38 and CD20 and digital image analyses (Immunoscore) of tumor specimens in an additional cohort of patients who received combined surgical resection and thermal ablation.

Results While comprehensive flow cytometric analyses in sequential samples (day 0, 7 and 90) of a prospective patient cohort $(n=23)$ demonstrated only moderate effects of MWA on circulating immune cell subsets, Fluorospot analyses revealed de novo or enhanced tumor-specific immune responses in 30\% of these patients. This anti-tumor immune response was related to tumor control. Interferon-y and Interleukin-5 $\mathrm{T}$ cell responses against cancer testis antigens were more frequent in patients with a long-time remission $(>12$ months) after MWA (7/16) compared to patients suffering from an early relapse (0/13 patients). Presence of tumor-specific $\mathrm{T}$ cell response (Interferon-y and/or Interleukin-5) was associated to longer progression-free survival (15.0 vs. 10.0 months). Immunohistochemical analyses of resected tumor samples revealed that a high $\mathrm{T}$ cell infiltration in a second tumor lesion at the time of thermal ablation was associated with superior disease-free survival (37.4 vs. 13.1 months).

Conclusions Our data demonstrates remarkable immune-related effects of MWA in HCC patients. This study and provides additional evidence for a combination of thermal ablation and immunotherapy in this challenging disease.
Funding 'Koeln Fortune' and 'CAP-CMMC' local research grant (to P.G. and H.A.S.) supported our research.

Disclosure Information E. Staib: None. K. Leuchte: None. M. Thelen: None. P. Gödel: None. A. Lechner: None. P. Zentis: None. M. Garcia-Marquez: None. D. Waldschmidt: None. R. R. Datta: None. R. Wahba: None. C. Wybranski: None. T. Zander: None. A. Quaas: None. U. Drebber: None. D.L. Stippel: None. C. Bruns: None. K. Wennhold: None. M. von Bergwelt-Baildon: None. H.A. Schlösser: None.

\section{P02.04 ABSTRACT WITHDRAWN}

\section{P02.05 INVESTIGATION OF A SYNGENEIC A-PD-1 ANTIBODY TO TREAT MURINE 4T1 MAMMARY CARCINOMA}

II Skandorff Pedersen ${ }^{*},{ }^{2} \mathrm{~K}$ Orfin, ${ }^{2} \mathrm{KN}$ Nielsen, ${ }^{2} \mathrm{PJ}$ Holst. ${ }^{1}$ University of Copenhagen, København N, Denmark; ${ }^{2}$ InProTher Aps, København N, Denmark

\subsection{6/jitc-2020-ITOC7.40}

Background Many cancers acquire mechanisms to evade immunosurveillance by activating immune checkpoint pathways, which suppress the antitumor immune responses. Monoclonal antibodies (ab's) targeting immune checkpoints, such as CTLA4 and PD-1, have shown excellent results in several cancers and are currently being investigated in clinical trials for various malignancies. The clinically tested a-CTLA-4 (Ipilimumab) and a-PD-1 (Nivolumab and Pembrolizumab) ab's are fully human or humanized ab's, respectively. However, most studies conducted in mice utilize a xenogeneic a-PD-1 ab originating from rat, IgG2a RMP1-14 clone. This has been proposed to cause adverse effects in the commonly used 4T1 mammary carcinoma model of triple negative breast cancer (TNBC). Repeated administration of xenogeneic a-PD-1 ab's in this model results in fatal hypersensitivity reactions in tumor bearing mice, and unlike human TNBC, the 4T1 cell line is generally poorly responsive to immune checkpoint inhibitors. Recently, a semi-syngeneic recombinant a-PD-1 ab has been developed by transferring the variable regions of RMP1-14 onto a murine IgG1e3 constant region.

Materials and Methods Testing xenogeneic and semi-syngeneic a-PD-1 ab with and without a-CTLA-4 ab in BALB/c mice carrying 4T1 luciferase positive tumors.

Results In this study, we compared a semi-syngeneic recombinant a-PD-1 ab to the original xenogeneic RMP1-14 clone for treatment of luciferase positive 4T1 carcinomas. Surprisingly, the semi-syngeneic a-PD-1 ab was not able to circumvent the fatal hypersensitivity reactions. Still, the combination therapy of a-CTLA-4 and the semi-syngeneic a-PD-1 ab significantly reduced tumor volume in 4T1-luciferase tumor bearing mice compared to isotype control-treated mice already from day 16 post tumor inoculation (day 8 post treatment-initiation). In contrast, xenogeneic a-PD-1/a-CTLA-4 treated mice did not show significant difference from the control group until 24 days post tumor inoculation and never to the same degree. Furthermore, analysis of the $\mathrm{T}$ cell responses towards the murine tumor-associated antigen $\mathrm{AH}-1$, revealed that treatment with syngeneic a-PD-1/a-CTLA-4 ab gave a significantly stronger $\mathrm{CD}^{+} \mathrm{T}$ cell response over both control mice and mice treated with xenogeneic a-PD-1/a-CTLA-4 ab. 
Conclusions These studies indicate that the semi-syngeneic aPD-1 IgG1e3 ab might be a more efficient and translatable aPD-1 ab for preclinical in vivo studies, which is important for the future investigation of immune checkpoint inhibitor therapy.

Disclosure Information I. Skandorff Pedersen: A. Employment (full or part-time); Significant; InProTher Aps. K. Orfin: A. Employment (full or part-time); Significant; InProTher Aps. K. N. Nielsen: A. Employment (full or part-time); Significant; InProTher Aps. P.J. Holst: A. Employment (full or part-time); Significant; InProTher Aps. E. Ownership Interest (stock, stock options, patent or other intellectual property); Significant; InProTher Aps.

\section{P02.06 ABSTRACT WITHDRAWN}

\section{P03 Tumor Microenvironment}

\section{P03.01 PREVALENCE OF CD112R+IMMUNE CELLS IN NORMAL LYMPHATIC TISSUES, INFLAMMATION AND THE CANCER MICROENVIRONMENT}

NC Blessin*, T Mandelkow, E Bady, C Hube-Magg, R Simon, G Sauter, C Fraune, M Lennartz, K Möller, D Höflmayer, SA Weidemann. University Medical Center HamburgEppendorf, Hamburg, Germany

\subsection{6/jitc-2020-ITOC7.41}

Background CD112R is an inhibitory immune checkpoint receptor and a putative target for novel immune therapies, but little is known about its molecular epidemiology in healthy and diseased tissues.

Materials and Methods To study the prevalence and expression level of CD112R ${ }^{+}$immune cells, we analyzed more than 200 samples of normal lymphatic, inflamed and cancerous tissues in a microenvironment tissue microarray format $(4 \mathrm{~mm}$ tissue spot diameter) and large sections using fluorescent multiplex immunohistochemistry.

Results CD112R expression was detected at variable intensity levels in $47 \%$ of $\mathrm{CD}^{+}$cytotoxic lymphocytes, $49 \%$ of $\mathrm{CD}^{+}$ $\mathrm{T}$ helper cells, $30 \%$ of $\mathrm{FOXP}^{+}$regulatory $\mathrm{T}$ helper cells and in $25 \%$ of $\mathrm{CD}_{5} 6^{+}$natural killer cells, but no expression was seen in $\mathrm{CD} 11 \mathrm{c}^{+}$dendritic cells and $\mathrm{CD} 68^{+}$macrophages. All analyzed compartments across normal and diseased tissues showed a small subset (CD8: 9 $\pm 18 \%$, CD4: 5 $\pm 15 \%$, FOXP3: $2 \pm 5 \%)$ of immune cells with supramaximal CD112R expression. The highest fraction of cells with supramaximal CD112R expression was found in the subset of $\mathrm{CD}^{+}$cytotoxic $\mathrm{T}$ cells in the Peyer's patches of ileum (62\%), the intergranuloma area of lymph node sarcoidosis $(27 \%)$ and in ovarian cancer (37\%). In cancerous tissues, the density and the fraction cytotoxic $\mathrm{T}$ cells with supramaximal CD112R expression was highly variable and ranged from $5 \%$ in bladder cancer to $3 \%$ in lung cancer and $36 \%$ in ovarian cancer. A high variability of the number of cells with supramaximal CD112R expression was also seen within every tumor entity.

Conclusions In summary, our analysis shows that CD112R expression is abundant in various subsets of immune cells but identifies a small fraction of cells with exceedingly high CD112R levels. The widespread occurrence of CD112R ${ }^{+}$ cytotoxic $\mathrm{T}$ cells in the cancer microenvironment may suggest considerable opportunities for checkpoint inhibitors targeting CD112R.

Disclosure Information N.C. Blessin: None. T. Mandelkow: None. E. Bady: None. C. Hube-Magg: None. R. Simon: None. G. Sauter: None. C. Fraune: None. M. Lennartz: None. K. Möller: None. D. Höflmayer: None. S.A. Weidemann: None.

\section{P03.02 SUPPRESSION OF T-CELL PROLIFERATION AND CYTOKINE RELEASE BY THE ADENOSINE AXIS ARE MEDIATED BY DIFFERENT MECHANISMS}

J Festag*, T Thelemann, M Schell, S Raith, S Michel, R Klar, F Jaschinski. Secarna Pharmaceuticals GmH and Co. KG, Planegg/Martinsried, Germany

10.1136/jitc-2020-ITOC7.42

Background The so-called adenosine axis has emerged as a promising therapeutic target pathway as high adenosine levels in the tumor microenvironment contribute to the suppression of antitumor immune responses. The ectonucleotidases CD39 and CD73 act in concert to degrade extracellular immunestimulating adenosine triphosphate (ATP) to immunosuppressive adenosine. According to the current model, subsequent suppression of effector immune cell function is caused by binding of adenosine to adenosine receptors like the A2a receptor (A2aR). The ectonucleotidases CD39 and CD73 as well as the $\mathrm{A} 2 \mathrm{aR}$ have emerged as molecular targets within the adenosine axis with currently more than 20 clinical trials investigating antitumor effects of CD39-, CD73- or A2aR blockade. We aimed to perform a direct comparison of these targets with regard to their roles in regulating T-cell proliferation and IFN- $\gamma$ secretion.

Materials and Methods CD39 and CD73 expression was suppressed using LNAplus ${ }^{\mathrm{TM}}$ antisense oligonucleotides (ASOs). ASOs were synthesized as gapmers with flanking locked nucleic acids (LNA) to increase stability and affinity to the target RNA, leaving a central gap for recruitment of the RNAdegrading enzyme RNaseH I. Knockdown efficacy of ASOs on mRNA and protein level was investigated in primary human $\mathrm{T}$ cells. Furthermore, the effects of ATP, AMP and adenosine analogues on $\mathrm{T}$-cell proliferation and IFN- $\gamma$ secretion were investigated. A2aR was blocked using small molecule inhibitors that are currently under clinical investigation.

Results Treatment of human T cells with LNA-modified ASOs specific for human CD39 and CD73 resulted in potent target knockdown in vitro without the use of a transfection reagent. T-cell proliferation was reduced after addition of ATP to activated $\mathrm{T}$ cells that was completely reverted by ASO-mediated suppression of CD39 and/or CD73 expression but not A2aR inhibition. Adenosine analogues inhibited IFN- $\gamma$ secretion of activated $\mathrm{T}$ cells, however, they did not suppress T-cell proliferation. Blockade of the adenosine kinase was able to revert the anti-proliferative effect of ATP degradation products, arguing for downstream metabolites of adenosine, but not $\mathrm{A} 2 \mathrm{aR}$ signaling, being responsible for the suppression of T-cell proliferation.

Conclusions Cytokine secretion and proliferation of $\mathrm{T}$ cells might be differentially regulated by the adenosine axis. Adenosine might primarily affect cytokine secretion via $\mathrm{A} 2 \mathrm{aR}$ signaling, whereas adenosine metabolites might especially impair proliferation of activated $\mathrm{T}$ cells independent from $\mathrm{A} 2 \mathrm{aR}$ 